\title{
Beiträge zur Anatomie und Physiologie der Sexualzellen und der Keimeinnistung beim Menschen.
}

\author{
Von \\ Hans Friedenthal (Charlottenbuig).
}

Die Sexualphysiologie weist besonders für den Menschen so schwerwiegende Lücken auf, daß eine Schilderung der wichtigsten Probleme und eine zusammenfassende Übersicht über unser gesichertes Wissen nicht überflüssig erscheint.

Wir besitzen bisher noch kein sicheres Merkmal, um die menschlichen Sexualzellenvorfahren so von den anderen Körperzellen zu unterscheiden, daß der Weg der Keimbahn von der befruchteten Eizelle bis zur ausgebildeten Sexualzelle lückenlos beschrieben werden könnte. Wenn. bei einigen Würmern die Keimbahnzellen durch ein Mehr an Kernschleifenmasse sich vom Entwicklungsbeginn an von den körperbildenden Zellen mit verminderter Kernschleifenmasse unterscheiden, so ist dies beim Menschen und den Säugetieren nicht zu sehen. Wohl zeigt die aufmerksame Betrachtung der ersten Zellteilung bei Säugetieren, daß die beiden ersten Furchungszellen bereits etwas verschieden sind und die Keimbahn sehr wahrscheinlich unter gewöhnlichen Verbältnissen einer der beiden Furchungszellen folgt, doch liegen noch keine ausreichenden Angaben über Keimbahnmerkmale bei Süugetieren vor. Durch Erhaltenbleiben der Körnerform in den Mitochondrien sollen später die Keimbahnzellen auch des Menschen sich von den anderen Körperzellen unterscheiden, und es werden Wanderzellen bei Menschenembryonen von 14 Ursegmenten als ersterkennbare Keimbahnzellen angesprochen, trotzdem sie mehrkernig sind. Sie fanden sich in der Gegend der Kloacke und unterschieden sich durch Größe und den Besitz von Dotterplättchen (?) von den übrigen Körperzellen. Es ist wahrscheinlich, daß das Studium des feineren Zellbaues bei der Mitose uns die Keimbahnzellen wird erkennen lassen. Während die Zahl der Kernschleifen in Keimzellen und körperbildenden Zellen die gleiche bleibt, entledigen sich die körperbildenden Zellen im Beginn der Entwicklung eines Teiles ihrer Kernschleifenmasse, so daß sichtbare Formverschiedenheit auftreten kann und die Keimbahn für das Auge des Beobachters sichtbar wird.

Verarmung an Kernschleifenmasse führt sehr allgemein die Zellen zu einer beschleunigten Reihe von Zellteilungen. Wir sehen die Keim- 
zellen hinter den körperbildenden Zellen an Teilungsgeschwindigkeit zurückbleiben, die Größe der Keimbahnzellen bleibt eine auffällige bis zur Bildung der kleinen Samenfäden nach der letzten Kernteilung. Die Gesamtentwicklung der Geschlechtszellen nimmt beim Menschen Jahrzehnte, in Ausnahmefällen mehr als ein Jahrhundert in Anspruch.

Die Vorstufen von Eizelle und Samenfaden gehören zu den kernstoffreichen und zugleich nährstoffreichen Entwicklungszellen. Wenn bei der ersten Furchung der befruchteten Eizelle bereits die grundlegende Scheidung in kleinere, schnell sich teilende und größere teilungsträgere Zellen einsetzt, folgt die Keimbahn dem Wege der größeren nährstoff- und kernstoffreichen Zellen. Bei einigen Tieren trennt die erste, bei einer Reihe von Tieren die dritte Furchung Zellen von ungleicher Größe und Kernbeschaffenheit. Die Keimlingszellen trennen sich in Dottergewebe und Bildungsgewebe, die zu verschiedenen Zeiten dem Dottergewebe entsprossen. In der Gegend des Urafters bleiben die Reste des Dottergewebes mit den Keimbahnzellen zu einer Zeit erhalten, wo der Keimlingsleib etwa Sandkorngröße und ein Alter von etwa 20 Tagen erreicht hat. Die Keimbahnzellen wandern zu dieser Zeit in die Bildungsstätten der späteren Eierstöcke und Hoden ein und werden dort als Ureier und Ursamenzellen durch ihre Größe und ihren Nährstoffreichtum deutlich von allen übrigen Körperzellen unterscheidbar, wenn der Keimling Haselnußgröße und ein Alter von etwa 30 Tagen erreicht hat. Nur etwa 25 Tage nimmt die Urgeschlechtszellenbildung bei beiden Geschlechtern in Anspruch, die durch eine geringe Zahl von Teilungen zu den Eimutterzellen und Samenmutterzellen führen. Beim Manne entstehen erst kurz vor der Geschlechtsreife, also über 12 Jahre nach der Geburt, die ersten Samenmutterzellen aus den Ursamenzellen. Im weiblichen Geschlecht findet beim Menschen im ersten Lebensjahre schon vereinzelt Eimutterzellenbildung, ja selbst Eizellenbildung statt. Die Ahnenstufen der Geschlechtszellen sind beim Mensehen wanderungsfähige, amöbenähnliche Zellen, welche an die wanderungsfähigen Eizellen der Schwämme und Hohltiere ebensowohl wie an die wanderungsfähigen Paarlinge der Einzeller erinnern.

Die Zellgröße der Keimbahnzellen nimmt von der befruchteten Eizelle bis zur Bildung der Ureizellen und Ursamenzellen ab, von da bis zur Bildung der Eimutterzellen und der Samenmutterzellen zu. Auf die Vermehrungsstufe folgt die Wachstumsstufe der Keimbahnzellen. In der Reifungsstufe bleibt die Größe der Eimutterzellen in der reifen Eizelle, der größten Zelle des Menschen, erhalten, während die Samenzellen sich ihrer Reservestoffe und ihres Plasmas entledigen und in die reifen Samenfäden übergehen, welche zu den kleinsten selbständigen Körpereinheiten zählen. Die Ähnlichkeit des Bildungsganges der männlichen und weiblichen Geschlechtszellen ist so groß, daß die Ursamen- 
zellen, obwohl Ahnenstufen der kleinen Samenfäden den Ureizellen. durchaus gleichend, wie diese die größten Zellen des Keimlings am Ende des ersten Entwicklungsmonats bilden, von allen Zellen der Eizelle am ähnlichsten geblieben sind.

Die Zahl der Zellteilungen, welche von Urei- und Ursamenzelle zu Eimutterzelle und Samenmutterzelle führen, ist gering. In der Reifungsperiode erfolgt in zwei Zellteilungen der Übergang zum reifen Ei und zu den Samenzellen, welche sich ohne weitere Teilung zu den Samenfäden umwandeln. Die Eireifungszeit währt vom ersten bis etwa ums 50. Lebensjahr, die Samenreifezeit vom 12. bis 70. Lebensjahr, in Ausnahmefällen bis zu dem höchsten beim Menschen beobachteten Lebensalter.

Der Mensch ist durch die Länge seiner Zeugungszeit, die abhängt von der Dauer der Reifungszeit der Geschlechtszellen, ausgezeichnet.

Die anfänglich äußere Gleichheit der Keimbahnzellen bei beiden Geschlechtern erinnert an die Zustände von annähernder Gleichheit der Paarlinge bei Einzellern, die große Zahl der Vorstufen der weiblichen Geschlechtszellen und der männlichen Geschlechtszellen an den Geschlechtszellenreichtum der auf äußere Befruchtung der Eier im Seewasser angewiesenen einfachen Lebewesen.

Die menschlichen Eierstöcke enthalten einige Jahre nach der Geburt mehr als eine halbe Million Keimbahnzellen, von denen etwa zweitausend im Laufe des Lebens zur Reife gelangen. Die Samenentleerung eines Mannes enthält etwa 200 Millionen. Samenfäden (60 Millionen im Kubikzentimeter). Im Laufe des Lebens könnte ein stets gesunder Mann bei 5000 Samenentleerungen eine Billion Samenfäden abgeben und damit der Fruchtbarkeit mancher Meerestiere nahekommen. Während ein Weib im Höchstfalle etwa dreißig ausgetragene Kinder zur Welt bringen kann, würde ein Mann bei stetem Wechsel einige Tausend Kinder zeugen können, immerhin nur einige Milliardstel der Zahl der von ihm erzeugten Samenfäden.

Die Keimbahnzellen, welche in die Geschlechtsleiste des jungen Keimlings einwandern, sind etwa dreißig Tage nach dem Beginn der Entwicklung als größere rundliche, hellere Zellen zwischen den kleineren, würfelförmigen Zellen der Kejmwülste zu erkennen, die männlichen, durch den Besitz einer unvollständigen Kernschleife von den weiblichen unterschieden. Eine bequeme Unterscheidung der Geschlechter wird erst möglich, wenn die Geschlechtswülste sich zu einem Hoden oder einem Eierstocke ausbilden. Die Ausbildung von Eifollikeln oder Hodenkanälchen hängt von dem Geschlecht der einwandernden Keimbahnzellen ab. In sehr seltenen Fällen wurde beim Menschen die Anlage einer Zwitterdrüse beobachtet, die aber stets nur eine Art der Geschlechtszellen zur vollen Reife ausbildete. 
Die Ursamenzellen sind große Zellen mit großem, kugelförmigem Kern, in welchem bis zu drei große Kernkörperchen neben reichem Netzwerk zu sehen sind. Den Kern umgibt eine dichte Masse, welche zwei kleinste Teilungskörperchen enthält. Zahlreiche Fädenkörnchen sind im Zelleib verteilt. Die Stützzellen und Ursamenzellen füllen die Samenkanälchen anfänglich aus, so daß erst später ein fortlaufender Hohlraum sich in ihnen bildet. Ganz allmählich wächst in etwa $13 \mathrm{Jahren}$ der Hoden bis zur beginnenden Geschlechtsreife heran, ohne daß eine erhebliche Zahl von Zellgeschlechtern von den Keimbahnzellen gebildet wird. Die letzten Abkömmlinge der Ursamenzellen, die Samenmutterzellen, sind kenntlich durch ihre Größe und das Verhalten ihrer Kernschleifen.

Im männlichen Geschlecht findet sich nur für kurze Zeit eine Andeutung der im weiblichen Geschlecht so ausgesprochenen Wachstumszeit der Keimbahnzellen. Auch hier nimmt die Zellgröße kurz vor den Reifeteilungen noch zu. Die Samenmutterzellen sind größer als die vorhergehenden Zellgeschlechter.

Die beginnende Reifung der männlichen Keimbahnzellen deutet sich durch Veränderungen des Aussehens der Kerne an. Die Kernschleifenmassen häufen sich an einem Pol des Kernes an unter Aufhellung der übrigen Kernräume. Es bildet sich ein Fadenknäuel aus, der durch einen doppelten, mehrfach abgeteilten Faden gebildet wird. Wenn die Samenmutterzelle sich zur Teilung anschickt, bildet sie nicht wie ihre Ahnenstufen bisher $23^{1}$ ) vollständige und I unvollständige Kernschleife aus, sondern nur 12 Kernschleifen, die vierfach erscheinen und die doppelte Stoffmasse enthalten wie die bisherigen Kernteilungsformen. Etwa viermal so viel wie die Kerne der Körperzellen. Die Zahl der Kernschleifen ist auf die Hälfte herabgesetzt. Bei der Teilung der Samenmutterzelle trennen sich die Vierergruppen der Kernschleifen zu je zwei Doppelgruppen. Die entstehenden Vorsamenzellen teilen sich bald darauf wieder in je zwei Samenzellen unter erneuter Trennung der Doppelschleifen. Aus einer Samenmutterzelle sind durch zwei Teilungen vier Samenzellen entstanden, welche die halbe Schleifenzahl und die halbe Kernschleifenmasse enthalten gegenüber den bisherigen Keimbahnzellen. Von den vier aus einer Samenmutterzelle entstandenen Samenzellen besitzen zwei eine unvollständige Kernschleife neben elf vollständigen, während die zwei übrigen zwölf vollständige Kernschleifen enthalten. Die ersteren bilden männchenbildende, die letzteren weibchenbildende Samenfäden aus. Durch Eindringen eines Samenfadens mit elf vollständigen Kernschleifen in eine Eizelle entsteht ein mannbildendes $\mathrm{Ei}$, während beim Eindringen eines Samenfadens mit zwölf vollständigen Kernschleifen in eine Eizelle ein weibbildendes Ei entsteht.

1) Die Zahl ist beim Menschen bisher nicht mit Sicherheit bekannt. 
Die Stufe der Zusammenlegung der Kernschleifen in den Samenmutterzellen mit der Bildung von Vierergruppen zeigt uns die Zusammensetzung unserer Erbmasse, die wir von unseren Eltern überkommen haben. Jedermann ist ein Bastard ron Vater und Mutter. Jede Samenmutterzelle zeigt in ihrer geschlechtsübertragenden Kernschleife die unvollständige Kernschleife des Vaters und die vollständige Kernschleife der Mutter in gespaltenem Zustande, so daß eine Viererbildung zustande kam. Durch die Vierzellenbildung bei den zwei Reifeteilungen wird bewirkt, daß die Samenzellen und die daraus sich bildenden Samenfäden nur die Hälfte der väterlichen Erbmasse mit der Hälfte der Kernschleifenzahl bei der Befruchtung übertragen. Ohne die Hälftelung der Erbmassen bei Ei und Samenfaden müßte beim neuen Geschlecht eine Verdoppelung der Erbmasse und der Kernschleifenzahl stattfinden und ein jedes geschlechtlich erzeugte Lebewesen die Eigenschaften aller vier Großeltern in bezug auf dasselbe Merkmal in sich vereinigen. Bei der Reifeteilung wird durch die Zusammenlegung der zusammengehörigen Kernschleifen und Spaltung derselben in Vierergruppen vor der ersten Reifeteilung bewirkt, daß die verschiedenen Anlagen für dasselbe Merkmal auf verschiedene Geschlechtszellen verteilt werden. Je abweichender die vier Großeltern eines Lebewesens gebaut sind, desto notwendiger ist die'gleichmäßige Verteilung bei der Reifung auf die verschiedenen Geschlechtszellen. Schon das Zusammentreffen zweier abweichender Erbmassen für dasselbe Merkmal kann Entwicklungshemmung zur Folge haben, die sich am frühesten bei der Bildung der Geschlechtszellen bemerkbar macht. Bei Bastardierungen sind häufig die entstehenden, anscheinend vollkommen ausgebildeten Bastarde unfruchtbar. Die Zusammenlegung der Erbanlagen vor den Reifeteilungen in den Zellkernen erfordert Ähnlichkeit der Paarlinge.

Nach Ablauf der beiden Reifeteilungen ähneln die vier neu entstandenen Samenzellen den früheren Keimbahnzellen, ihre Größe beträgt nur ein Viertel der Samenmutterzellen. Durch eine Reihe von Umbildungen geht aus allen vier Samenzellen je ein Samenfaden hervor. Der Kern wird dichter, kleiner und eiförmig durch Verlust des Kernsaftes. Der Kopf des Samenfadens geht aus dem Kern hervor und erhält eine Kopfkappe mit Schneideapparat aus der starken Hülle der kJeinen Teilungskörperchen. Die Fadenkörnchen und Teilungskörperchen bilden ein Mittelstück, welches von verschiedenen, meist aber geringen Protoplasmamengen umbüllt wird. Aus den Teilungskörperchen sproßt eine elastische Fibrille, welche bis auf das äußerste nackte Ende mit einer dünnen Plasmascheide umhüllt wird und den Schwanz des Samenfadens bildet. In wenigen Monaten hat sich unter Erhaltung der Kernschleifenmasse, der Teilungskörperchen, der Fadenkörnchen und eines geringen Plasmarestes die männliche Geschlechts- 
zelle von allem befreit, was ihr bei der Aufsuchug der befruchtungsbedürftigen Eizelle hinderlich sein könnte und in Schwanzruder und Bohrkopf Bewegungsapparate ausgebildet, welche ihr Aussehen dem eines Geißeltierchens ähnlich machen. Da den Samenfäden Nährmaterial nur in geringer Menge zu Gebote steht, senken sie sich mit dem Kopf voran in den Plasmaleib der Hodenstützzellen, welche Stoffzufuhr zu den Samenfäden vermitteln. Nach Ausstoßung und Ablösung aus den Hodenstützzellen können die jungen Samenfäden noch viele Monate im Nebenhoden verweilen, fallen aber Rückbildungsvorgängen anheim, wenn ihre Ausstoßung gar zu lange auf sich warten läßt.

Der reife menschliche Samenfaden mit eiförmigem Kopf von der Fläche gesehen, mit spindelförmigem Kopf von der Kante gesehen, ähnelt in hohem Maße den Samenfäden der Menschenaffen, in etwas geringerem Grade den Samenfäden der niederen Affen und einiger Halbaffen. Alle übrigen Tiere und Pflanzen besitzen abweichend gebaute Samenfäden.

Wenn zwei Lebewesen zugleich im Bau der Eier und Samenfäden große Ähnlichkeit zeigen, brauchen wir an ihrer natürlichen Verwandtschaft nicht mehr zu zweifeln. Eine auffällige Ủbereinstimmung zweier Lebewesen nur in der Form der beiden Geschlechtszellen ohne sonstige Hinweise auf nahe Verwandtschaft ist nicht bekannt.

Die Samenfäden des Menschen besitzen eine lang anhaltende Eigenbeweglichkeit. Die peitschenden Bewegungen des Schwanzes treiben den Kopf mit einer Geschwindigkeit von etwa $0,006 \mathrm{~cm}$ in der Sekunde vorwärts. Die Samenfäden stellen sich mit den Köpfen gegen die Strömung, sie vermögen erheblich größere Zellen durch ihre Bewegung beiseite zu schieben. In Leichen vermögen Samenfäden sich tagelang beweglich, in den weiblichen Geschlechtsteilen wochenlang, bei Fledermäusen mehr als sechs Monate lang lebendig und zeugungsfähig zu erhalten. Bewegungslos liegen die Samenfäden in den Nebenhoden. In der Samenflüssigkeit, die vor allem von den Drüsen der männlichen Geschlechtsorgane geliefert wird, erwacht die Beweglichkeit der Samenfäden und kann noch außerhalb des Körpers über eine Woche lang erhalten werden. Die Flimmerfortsätze in den weiblichen Ausführungsgängen erzeugen in Eileiter und Gebärmutter eine schwache Strömung nach außen, welche die Samenfäden nach dem Eierstock hinleitet. Das Einbohren der Samenfäden in die Eihülle des menschlichen Eis braucht nicht durch abgesonderte Stoffe von seiten des Eis ermöglicht zu werden. Bei zahlreichen Lebewesen werden die Samenfäden durch chemische Stoffe zum Ei geführt und dort durch besondere Pforten der Eihüllen zum Eiprotoplasma hingeleitet, nicht aber bei dem an sich nackten Menschenei, welches von den in Auflösung begriffenen Begleitzellen wie von einer besonderen Hülle umgeben wird. 
Die Aufgabe der Samenfäden, die Kernschleifenmasse und die Teilungskörperchen in das Plasma der weiblichen Eizelle einzuführen, wird zwar von den Samenfäden der Lebewesen gelöst, doch weicht der Bau der verschiedenen Samenfäden oft weit ab von dem Bau eines Bohrers, der in reinster Form sich in den Samenfäden der Finkenvögel verwirklicht findet. Die menschlichen Samenfäden haben eine meilielartige Kopfkappe, die bei den beständigen Drehungen recht erhebliche Reibungswiderstände verursacht. Die Fortbewegung des Kopfes durch die Schwanmbewegungen erfolgt nach Art eines Bootes, welches durch seitliche Bewegungen eines in der Mittellinie hinten angebrachten Ruders vorwärts getrieben wird (Wricken). Mit dieser einfach erseheinenden Vorrichtung vermögen die Samenfäden in acht Tagen einen Weg von $50 \mathrm{~m}$, also das Millionenfache ihrer eigenen Länge zurückzulegen, während unsere Dampfer noch nicht das Hunderttausendfache ihrer Lünge, ohne Kohlen zu fassen, zurückzulegen vermögen, also noch nicht ein Zehntel dieses Weges. Die Samenfäden sind außerordentlich wirtschaftlich gebaute Bewegungsmaschinen. Das Gewicht der Bewegungsmaschine und des Heizmaterials zusammen ist derart gering gegenüber dem Weg und der abzuliefernden Last, dal.s unsere heutige Technik auch nicht einmal Ähnliches zu leisten imstande ist.

Die chemische Zusammensetzung der Samenfäden ähnelt mehr der Zusammensetzung von Zellkernen als der von Körperzellen. Besonders auffällig ist dio Wasserarmut der Samenfäden und der dadurch bedingte hohe Gehalt an Salzen und Kornstoffen. Durch Zusammen(Irängen der zu befördernden Stoffmasse auf denkbar kleinstem Ramm wirll die Bewegungsleistung erleichtert. Durch Wasserzufuhr wird aus dem Samenfadenkopf ein großer \%ellkern nach dem Eindringen in die Wizelle neu gebildet.

Einen für unsere Vorstellung langen Weg haben die Keimbahnzellen des männchenbildenden Eies bis zur Bildung der zeugungsfähigen Samenfäden zurüekgelegt. Alle Umwandlungen dienten der Aufgabe, eine selbstbewegliche Maschine zu schaffen, welche die Hälfte der ererbten Kernsehleifenmasse und ein Teilungskörperchen in dem Inneren einer befruchtungsfähigen Eizelle abzuliefern imstande ist. Wir kömnen schätzen, daß nur einige Billionstel der erzeugten mensehlichen Samenfäden zur Erzeugung eines Menschen verwandt werden, während allo iibrigen als Abfallstoffe im Haushalte der Natur Verwendung finden.

Die Keimbahnzellen der weibchenbildenden menschlichen Eier mit 24 vollständigen Kernsehleifen verhalten sich bis zur Ureierbildung wio die der männchenbildenden Eier mit 23 vollständigen Kernschleifen. Wie bei diesen spalten nach den ersten Furchungen die Keimbahnzellen Zellen von sich $a b$, deren Kernschleifenmasse sich verringert und die daraufhin zu rascherer Teilung befähigt, den weiblichen Körper 
aufbauen als Träger der langsamer sich teilenden und weniger verändernden Keimbahnzellen. Wie beim männlichen Geschlecht wandern Keimbahnzellen von der Gegend der Kloakenhaut (dem späteren After) in die Geschlechtsleisten ein, welche sich aus dem Mittelblatt etwa 25 Tage nach dem Beginn der Entwicklung angelegt hatten. Während die Körperzellen die Fadenkörnchen in ihrem Plasma in Stäbchen und Fäden umwandeln, bleiben diese bei den Keimbahnzellen in einzelnen Körnern erhalten, so daß eine Unterscheidung zwischen Keimbahnzellen und Körperzellen möglich ist.

Die Urnierengeschlechtsfalte legt sich zu beiden Seiten der späteren Wirbelsäule in dem Raum hinter der Leibeshöhlenwand an und drängt deren Wandung vor sich her bei Keimlingen mit einigen 20 Körperkästchen. Der Weg der Keimbahnzellen bis zu ihrer Einnistungsstätte, den diese wanternd zurückzulegen haben, ist nicht lang wegen der geringen Größe des Keimlings, doch reicht die Keimdrüsenanlage vom sechsten Brustkästchen bis zum zweiten Kreuzkästchen hinunter.

Auf die Einwanderung der Keimbahnzellen in die Geschlechtsleisten folgt die Vermehrungszeit, welche zu einer raschen Größenzunahme der sich ausbildenden Eierstöcke führt unter gleichzeitiger Ausbildung von Bindegewebe und Blutgefäßen im Eierstock. Ohne scharfe Grenze geht die Vermehrungsstufe der Keimbahnzellen in die Wachstumsstufe über, und sehon vom hundertsten Tage nach der Befruchtung ab setzt auch schon die Auflösung und Rückbildung zahlreicher Keimbahızellen. ein, die von da ab ununterbrochen zunimmt, bis gegen das 50. Lebensjahr hin keine Keimbahnzellen mehr im weiblichen Körper anzutreffen. sind.

Die weiblichen Keimbahnzellen besitzen nicht nur mehr Kernschleifenmasse als die weiblichen Körperzellen, sondern auch mehr Protoplasma. Durch ihr helles Aussehen, durch ihre Größe, ihren großen Zellkern mit weitmaschigem Kernnetz und Fehlen von Kernkörperchen sind sie von den Körperzellen der Eierstöcke zu unterscheiden. Sobald die Keimbahnzellen von Körperzellen rings umgeden sind, die Eierstockbläschenbildung begonnen hat, setzt die eigentliche Wachstumszeit der Keimbahnzellen ein. Nach dem dritten Lebensjahr findet keine neue Eierstockbläschenbildung mehr statt, sondern die Reifung der früher angelegten und die Rückbildung der übergroßen Mehrzahl. Die in den letzten Schwangerschaftsmonaten gebildeten Eierstockbläschen der Frucht haben neugebildet einen Durchmesser von etwa $0,05 \mathrm{~cm}$. Die Keimbahnzelle wächst bis zu einem Durchmesser von $0,025 \mathrm{~cm}$ im Eierstockbläschen unter reicher Aufnahme von Nahrungsmaterial heran, vermehrt also ihre Masse in der eigentlichen Wachstumszeit um das 125fache. Die Kernschleifenmasse nimmt an dem. Wachstum der weiblichen Keimbahnzelle nicht teil, nur die Größe des 
Zellkemes, so daß in der reifen Zelle der Kern als wasserhelles großes Bläschen sichtbar ist, ohne Fadenwerk mit großem Kernkörperchen.

Während der Wachstumszeit im Eierstockbläschen liegen die weiblichen Keimbahnzellen weit von allen Blutgefäßen entfernt und müssen Sauerstoff und Wachstumsbausteine durch die umbüllenden Zellen hindurch beziehen und ihre Ausscheidungsprodukte zwischen diese hinein absondern. Gegen Ende der Wachstumszeit löst sich der Eihügel ab und schwimmt frei im Eierstockbläschen umher, bis er beim Platzen desselben in die den Eierstock umfassende und ihm sich eng anlegende Eileitermündung entleert wird. Im Innern der wachsenden Eizolle findet sich neben dem Kern ein Dotterkern genanntes Gebilde aus dichterer Innenmasse und dicker Hüllmasse, die bei Reifung der Zelle, aufgelöst wird und schwindet. Wir können den Dotterkem dem 'T'eilungskörperchen der mämmlichen Keimbahnzellen mit seiner Hülle, die später die Kopfkappo des Samenfadens liefert, vergleichen. Die 'Teilungskörperchen der Eizelle gehen zugrunde, während durch den Samenfaden bei der Befruchtung ein sich verdoppelndes neues Teilungskörperchen eingeführt wird. Alle Zellen von Mann und trau besitzen nur vom Samenfaden, also vom Vater stammende Teilungskörperehen.

Durch Verquellung der an die Eizelle anstoßenden Bläschenzellen bildet sich eine durchsichtige Hülle um das wachsende $\mathrm{Ei}$, an deren Bildung die Ausscheidungsstoffe der Eizelle beteiligt sind. Diese Hülle, die bei den ersten Furchungen verloren geht, muß bei der Befruchtung von den Samenfäden, die sich zwischen die Begleitzellen des Eies gedräingt haben, durchbohrt werden.

Der Fidotter wird beim Menschen in kleinen Schollen und feinen Körnchen in der Umgebung des Eikernes abgelagert, während die Randräume in der Betrachtung von oben dotterärmer erscheinen. Bei den Wirbeltierciem ist ein Teil des Dotters schwerer, ein fetthaltiger Teil, der grobe Tropfen bilden kann, wie bei den Knochenfischen, leichter als der Eizellstoff.

Die Eireifung sorgt für Hälftelung der Kernschleifenmasse und gleichmäßige Verteilung der auszuscheidenden Erbanlagen. Während oder nach der Ausstoßung der Eimutterzelle mit ihrer Hülle und ihren Begleitzellen kann die Reifung erfolgen und braucht beim Eindringen des Samenfadens, der fast unmittelbar nach der Ausstoßung in dic Eizelle eindringen kann, noch nicht beendigt zu sein. Die Kernschleifen legen sich wie bei den Samenmutterzellen in den Eimutterzellen ancinander und bilden nach Spaltung Vierergruppen, zwölf an der Zahl, welche im Gegensatz zu den. Samenmutterzellen keine unvollstïndigen Kernschleifen aufweisen. Nach Bildung der Kernteilungsfigur spalten. die Vierergruppen sich in Doppelgruppen. Zwölf Doppelgruppen von Kernschleifen werden mit einer geringen Plasmamenge unterhalb der durchsichtigen Hülle von der Fizelle abgetrennt. Das abgetrennte 
Zellstück wird Richtungskörperchen oder Polzelle genannt. In einer zweiten Teilung trennen sich in Eizelle und Polzelle die Doppelgruppen von Kernschleifen. Es wird eine neue Polzelle mit zwölf einfachen Kernschleifen ausgestoßen. Nach Beendigung der Reifeteilungen liegen drei Polzellen mit je zwölf Kernschleifen zwischen Eizelle und Hülle. Der Kern der Eizelle enthält nur noch die Hälfte der Kernschleifenzahl, ebenso wie der Kern der Samenzelle nach der zweiten Reifeteilung. Vor der Eireifung muß die Eimutterzelle die doppelte Masse an Kernschleifensubstanz gehabt haben, damit nach der Vierzellenbildung die Hälfte der für die Keimbahnzellen üblichen Menge an Kernschleifenmasse übrigbleiben kann. Aus den zwölf übriggebliebenen Kernschleifen bildet sich in der reifen Eizelle ein neuer Kern, der weiblicher Vorkern genannt wird. Aus dem Kopf des eingedrungenen Samenfadens entsteht durch Flüssigkeitsaufnahme aus der Eizelle ein Kern, der männliche Vorkern, der bis zur Größe des weiblichen Vorkernes heranwächst, auf diesen zuwandert, sich an ihn anlegt und zuletzt mit ihm verschmilzt. Der neue Kern, der erste Kern des neuen Geschlechts, entspricht einem Kern mit 24 Kernschleifen, wenn ein weibchenbildender Samenfaden eingedrungen war, von denen zwölf den beiden Großeltern von Vaterseite, zwölf den beiden Großeltern von Mutterseite her ihrer Herkunft nach entsprechen. Drang ein männchenbildender Samenfaden ein, so enthält der erste Kern des neuen Geschlechts, wie alle folgenden, eine unvollständige Kernschleife neben 23 vollständigen. Das doppelte Teilungskörperchen der neuen Zelle stammt aus dem Mittelstück des eingedrungenen Samenfadens.

Zwei Keimbahnen laufen bei der Befruchtung zusammen und bilden eine neue, in ganz genau gleicher Weise noch nie in der Welt dagewesene und nie in genau gleicher Weise wiederkehrende Zusammenstellung von Erbanlagen.

Da alle Lebewesen miteinander verwandt sind, d. h. gemeinsame Ahnenstufen besitzen, so sind erst recht die Keimbahnen zweier befruchtungsfähiger Paarlinge vielfältig in der Vergangenheit miteinander verknüpft und verschmolzen. Wenn die Maschen des Netzes der Keimbahnen dicht hinter den Paarlingen zusammenschließen, sprechen wir von Inzucht oder Verwandtenpaarung, wenn eine Reihe von Geschlechtern bis zur früheren Kreuzungsstelle der Keimbahnen zu verfolgen ist, sprechen wir von Fremdbefruchtung oder Bastardbildung. ${ }^{1}$ )

1) Die Netzbildung der Keimbahnen der Ahnenstufen erklärt die Schwierigkeit einer befriedigenden Einordnung der Lebewesen nach ihrer Abstammung. Eine auf die tatsächlich beobachteten Verschiedenheiten der Lebewesen aufgebaute (künstliche) Ordnung erlaubt eine raschere und bequemere Auffindung als eine auf den Verwandtschaftsverhältnissen aufgebaute natürliche Ordnung, deren Aufsuchen dem, der die Natur kennen lernen und mit dem Geiste nachschaffen will, unentbehrlich ist. 
Bei der: Befruchtung der menschlichen Eizelle fiel bereits dic Entschcidung, ob ein Mann oder ein Weib erzeugt werden wird, je nach der Zahl der vollständigen Kernschleifen in den Samenfäden. Das Geschlecht des künftigen Lebewesens ist nur eine der vielen Anlagen, welche bereits bei der Befruchtung vorher bestimmt sind durch dic Beschaffenheit des väterlichen und mütterlichen Erbgutes. Verläuft die Entwicklung in gewohnten Gleisen, so erscheint das Leben der Neuerzengten bis ins kleinste bereits vorgezeichnet und vorherbestimmt durch die Erbanlagenmischung. Bei Änderung der Unweltsverhältnisse lassen die Abweichungen vom ererbten Entwicklungswege erkennen, daß nur ein Teil dessen, was wir unser Schicksal nennen, in den Erbregeln zum Ausdruck kommt.

Der Beginn der Entwicklung des Menschen aus der Eizelle nach deren Vereinigung mit einem Samenfaden, noch niemals beobachtet, kann nicht erfahrungsgemäß geschildert, sondern nur aus Vergleichen mit dem Entwicklungsbeginn anderer Säugetiere erschlossen werden. Verhältnismäßig nahverwandte Säugetiere zeigen oft erhebliche Abweichungen in Form und Lebenswejse der ersten Lebensstufen. Die befruchtete Eizelle des Menschen kann mit unbewaffnetem Auge als allerkleinstes Kügelehen durch die Spiegelung an der gewölbten glasigen Oberfläche bei hervorragender Sehschärfe wahrgenommen werden. Gegen dunklen Hintergrund in der Sonne schimmernde allerfeinste 'Iautröpfchen an feinen Spinnenfäden geben cin zutreffendes Bild von dem Aussehen eben befruchteter, durch den Eileiter wandernder Säugeticreier. Bei etwa zehnfacher Vergrößerung erkennt man eine gekörnelte Innenkugel, die von einer schmalen, durchsichtigen, schleimigen Kapsel umhüllt wird und an ihrer Oberfläche ein kleines Bläschon mit noch viel kleinerer Innenkugel dei stärkeren Vergrößerungen erkennen lassen würde. Drei kleinste Kügelchen liegen zwischen Hülle und Innenkugel mit Kernelementen in ihrem Innern, ein kleines dichtes Pünktehen liegt jn der Nähe des Bläschens in der Innenkugel. Das Keimbläschen mit dem Keimfleck ist leichter als die halbflüssige Innenmasso der Wizelle, und auch die größeren Dotterkügelchen sammeln infolge Fettgehaltes sich am oberen Kugelabschnitt. Bei der Ansicht von oben liegt daher das Keimbläschen mit dem Keimfleck etwa in der Mitte der Eizelle, umgeben von einem breiten Hof von Dotterelementen, der umsäumt wird von einem breiten helleren Ring mit feineren Einschlüssen. Der Querschnitt der Dotterhaut umschließt als feine, dunklo Linie die Innenkugel. Außerhalb der Dotterhaut liegen die drei kleinen. Polzellen eng umschlossen von der Außenhülle, welche aus Zelltrümmern besteht, die durch Verdauungsfermente angegriffen und in Auflösung begriffen. sind. Nur von der Seite gesehen ist die Schichtung der Teile nach dem Raumgewicht, das Schwimmen des Kernes oder 
Keimbläschen sowie der grölßeren Dotterelemente an der Oberfläche zu erkennen.

Der Mensch beginnt seine Lebenslaufbahn mit der Kugelform, losgetrennt von jeder festen Verbindung mit den mütterlichen Teilen, in denen er gewachsen, die ihn aber nicht erzeugt hatten. Die Kugel besitzt von allen Körpern gleichen Rauminhaltes die kleinste Oberfläche. Je größer die Oberfläche eines Lebewesens .... und auch die befruchtete Eizelle ist ein einheitliches Lebewesen ......., desto größer die Einwirkungsfähigkeit der Umwelt, desto reicher die Beziehungen zur Außenwelt. Bei den ebenfalls annähernd kugelförmigen, frei im Raume schwebenden Weltkörpern faßt eine dem Menschengeist erkennbare und berechenbare Gesetzlichkeit alle Körper zu einer Einheit zusammen und läßt jede Veränderung als vorausberechenbar und notwendig erscheinen, auch für die Lebewesen gibt es keine ungesetzliche, theoretisch unberechenbare Veränderungsfähigkeit oder eincn freien Willen auf irgendeiner Lebensstufe.

Die befruchtete Säugetierzelle besitzt durch ihre Kugelgestalt, ihre Vereinzelung, ihre Dotterhaut, die ihre lebendige Masse von del: Hülle und von der Außenwelt trennt, scheinbar in hohem Grade dic Fähigkeit, auf sich selber gestellt in Ruhe zu verharren. Zahlreicho Einzeller und Pflanzen besitzen abgekapselte Sporen als Ruhestufon, welche den Eizellen ähneln. Tatsächlich verlaufen auch die Lebens. prozesse im allerersten Lebensanfang mit einer im späteren Leben ungewöhulichen Langsamkeit in den befruchteten Eizellen. Die Stoffaufnabme und -abgabe - Nahrungsaufnahme, Verdauung und Ausscheilungen - ist anfänglich bis auf einen unerkembaren Rest verkleinert. Die Atmung wird nicht nur durch die Kleinheit der Austauschflächo bei der Kugelgestalt, sondern auch durch die räumliche Trenmung von len sauerstofführenden. Blutgefäßen der mütterlichen Teile stark bohindert und damit auch der Kraftwechsel auf kleinste Werte herabgedrückt. Ein äußeres Wachstum im Sinne der Massenzunahme findet beim Menschenei anfänglich kaum statt, sogar eine gewisse Verkleinerung durch Abstreifung oder Auflösung der Hülle, welche während der Befruchtung das̀ $\mathrm{Fi}$ umga:b und seinen Durchmesser vergrößern half.

Nur eine einzige, allen Lebewesen eigene Betätigungsform erscheint gesteigert in der befruchteten Eizelle, die Vermehrungsfähigkeit. Der Kern einer Zelle dient dem Stoffwechselbedürfnis der Zelle. Je größer die Menge an Zellstoff in einer Zelle, desto weiter die Wege bis zum Kern und desto schwieriger die Versorgung mit lebensnotwendigen Stoffen and die Verwertung derselben. Die Eizelle besitzt viel Zellstoff und verhältnismäBig wenig Kernsubstanz. Durch die bei der Zellteilung sich abspielenden Vorgänge wird bei gleichbleibender Masse 
in Kensubsanz die Kemobertläde, die Austauschflächo zwischen Ken and Zellstoff, vergeöhert, moist gleichzeitig auch die stoffaufnehmonde: zolloberfláche.

Die Versohmotzung von mämblehen und weiblichem Kern bei der

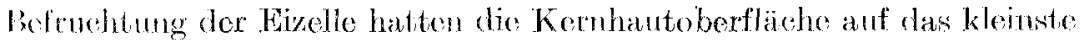
mögliche MaB horabgemindert. Dareh Kellvormehrugg wirl nash kuryer Zacit die Sumuno der Kernobotlächen vergrößert und spätor duroh $\Lambda$ ufyabo der Kugelgestalt die Leibesoberfähe vermehrt. To einfachsto losiung ar Aufgabe -...- orleiehterte Stoffaufuhr ohne Aufgabe dor Kugelgestalt -.. dureh cino VergröBerung der Kugebobertäehe un! Anordnung dor stoffwohselberiürtigen Masso in einer vergröberten Kugeloberfiache kamn nur bei gleichzoitger Vergröberung der Kern abortläe Hilfo bringen.

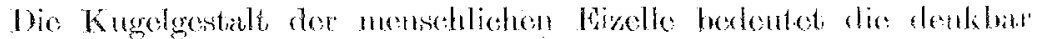

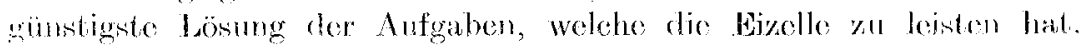
bie kizolle hat wio der Samenfaken eine gewisse Menge an Komwhloifensubstanz aufzubewahren und die Vereingung mit dem Samenfaden zu ermöglichen. Da dio zu übertragende Kenschleifonmasse; woch nieht ein Tausendstol des Figewichtes ausmacht, wäre zur lösung

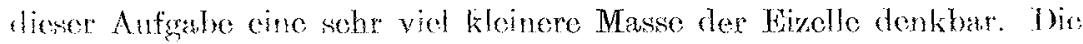
Wizclle hat die weitore Aufgabe, die doppelte Mengo Kellstoff, als ihrem. Gewicht an Kernsehleifenmasse entspricht, als Ersatz für dic vertorengegangene Plasmamengo des Samenfadens aufaupoichom wnel (a) iaber hinaus so viel an Nïhrstoffen, dall ohno Aufuahmo wesent lichor Mengen neuer Nahrung cin selbsternihurugsähiger Keimling

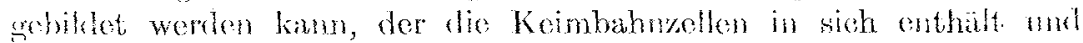
weiterfuhrt. Die Cestalt der Eizelle muß die Weiterbefördermeg clureh

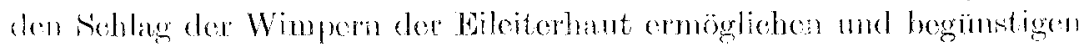
und thre Grobe die Auffindung darch die Samenfäden erleichtern. Bo fruchtung des lis folgt ter Begattung bei den Tieren mit innerer Bo. salmung dos Woibehens in ganz verschiedence \%eitfolge. Bei vielon Tioren

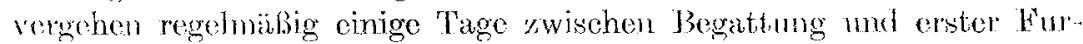
ahung, bo Fledermänsen findet dio Begattung im Herbst, die Eibefruch tung in Fröhjahr stat, bei den Bienen kann die Eibefruchtung einge: habre nach der nur cinmal im Loben stattindenten Besammig orfolgen. Boim Monschen soheint dic Befruchtung in einer gewissen Abhängigkoit vou der lireifung und von dem Zeitraum der Begattung zu stohen, zu. weilen nach wenigen Stunden, zuweilen aber erst nach einer Boibe von Tagen auf die Besammg zu folgen. Im Gegensatz zu der Mehrzahl dor Tiero kann beim. Menschen zu jeder beliebigen Zeit erfolgte Besamung zur Eibefruchtung führen, wenn auch die Besamung kurz nach abgelaufoncr Monatsblutung weit häufiger zur Schwängerung führt, als eine kurz vor: (ler Monatsblutung erfolgte. Bei amorikanischen Negern erfolgt dic 
Begattung vorzüglich zur Zeit der Monatsblutung, weil alsdann der: stärkere Geruch des Weibes den Neger zur Begattung anregt. Bei der Mehrzahl der Säugetiere wissen die Weibchen zur Zeit der Brunst alle vorzeitigen Begattungsversuche der durch Riechnervenerregung frühzeitig begattungslustigen Männchen entweder mit Gewalt abzuschlagen oder sich ihnen zu entziehen.

Nach erfolgter Befruchtung setzt bei der Mehrzahl der Tiere rasch die Entwicklung des Keimlings ein, beim Reh kommt es allerdings recht bald zu einem Entwicklungsstillstand, auf den einige Monate später eine Zeit rascher Weiterentwicklung folgt. Die Tragzeit des Menschen von etwa 273 Tagen muß bei Berücksichtigung der Körpergröße des Menschen als lang bezeichnet werden, zumal in Anbetracht der Unbehilflichkeit und Unfertigkeit des menschlichen Neugeborenen. Die lange Tragzeit vieler Huftiere dagegen findet in dem hohen Ausbildungszustand des Neugeborenen ihre Erklärung.

Aus der Maulbeerlarve bildet sich ein Zellhaufen durch raschere Teilung der kleineren Zellen, bei welchem im Innern liegende größere dotterhaltige Zellen umwachsen sind von einer einschichtigen Hülle kleinerer Zellen. Die Keimbahnzellen liegen im Innern des Zellhaufens. Auf dieser Stufe, die bei sämtliehen Tieren der Bläschenstufe mehr oder weniger deutlich vorangeht, ähnelt der Menschenkeim gewissen Tieren, die in der Mitte zwischen Einzellern und vielzelligen Tieren stehen und deshalb Utbergangstiere oder Mesozoen genannt werden. Eine Höhlung mit flüssigem Inkalt braucht auf dieser Stufe beim Menschen nicht aufzutreten.

Sobald der Menschenkeim die Übergangstierstufe erreicht hat, beginnen Vorgänge, welche die Einnistung des Keimes in die Gebärmutterschleimhaut einleiten. Die Zellgrenzen der äußeren Schicht beginnen sich zu verwischen und die zusammengeflossene Zellmasse treibt selbstbewegliche Fortsätze nach Art der freilebenden Amöbentiere. Den Kernen in der zusammengeflossenen Zellmasse fehlt die Fähigkeit zur Fadenteilung. Der Keim besitzt die Fähigkeit, in die mütterliche Schleimhaut einzudringen, wobei rings um den eindringenden Keim eine Zerstörung und Auflösung der mütterlichen Gewebe sich zeigt, die an die Auflösung der Begleitzellen der ausgestoßenen Eizelle erinnert und wie diese auf einer Ausscheidung zellverdauender Säfte aus dem Keim in die Umgebung beruht. Auf ganz ähnliche Weise dringen bei Ruhr die großen Darmamöben in die Darmschleimhaut ein, unter Zerstörung und Auflösung der Gewebe. Der Menschenkeim ist mit allen Hilfsmitteln ausgerüstet, welche gewissen freilebenden Schmarotzern die Einnistung ermöglicht, und erzwingt sich auf diese Weise die Zufuhr von Nährstoffen von seiten der Mutter, wodurch seine Dotterarmut wettgemacht wird. 
Am Ende der ersten Lebenswoche ähnelt die Höhe der EntwickIung des Menschenkeims noch immer der von allereinfachsten Lebowesen, nämlich der von schmarotzenden Mesozoen. Ohne Ausbildung irgendweleher aus Zellen zusammengesetzter Organe hat eino Trennung stattgefunden zwischen Keimbahnzellen und Körperzellen. Die Körperzellen umgeben die innen liegenden Koimbahnzellen und haben die Aufgabe, für Ernährung, Atmung, Ausscheidung und Anheftung des Keimes zu sorgen. Die Zellgrenzen der Außenzellen verschwinden und es entsteht, eine einheitliche äußere Plasmaschicht mit zahlreichen Kernen. Durch selbstbewegliche Wurzelfüße, welche in die mütterlichen Gewebe einzudringen vermögen, wird die zelltrümmer fressende und verdauende Oberfläche vergrößert und ein Festhaften des Keimes ermöglicht. Der Menschenkeim setzt sich fest in einer Form, clic an die Anheftung schmarotzender Coelenteraten erinnert, bei denen es ebenfalls zu einem Zusammenfließen der ausläuferbildenden Zellen zu einer einheitlichen Protoplasmamasse kommt. Das anfängliche Fehlen eines Hohlraumes im sich festsetzenden Keimling, die Innenlagerung der Keimbahnzellen und das Zusammenfließen der Außenzellen sind Anpassungen an die schmarotzende Lebenswcise, für welche geringer Raumbedarf wesentlich ist.

Auf dreierlei Weise ist für die Ernährung und Erhaltung des neuen. Geschlechts bei den Lebewesen gesorgt. Auf der ersten Stufe werden (bie Eier oft noch vor der Befruchtung vom mütterlichen Organismus in die Umgebung ausgestoßen, ausgerüstet mit der Befähigung, sich sogleich selbständig ihre Nahrung zu erwerben.

Auf der zweiten Stufe bekommen die Eizellen einen Vorrat von Nähr-stoffen bei der Eiablage mitgeliefert, welcher ihnen crlaubt, auf selbstian(ligen Nahrungserwerb zu verzichten, bis die erreichte Entwicklungshöhe für selbständigen Nahrungserwerb ausreicht.

Auf der dritten Stufe, dio an die erste, nicht an die zweite, anknüpft, wird der Keimling befähigt, als selbständiger Schmarotzer seine Nahrung lem mütterlichen Leibe zu entnehmen. Diese dritte Stufe ist der zweiten. wirtschaftlich deshajb bedentend überlegen, weil nur der wachsende Keimling nach dem Ausmaße seiner Bedürfnjsse mit Nahrung versorgt zu werden braucht, während auf der zweiten Stufe unabhängig, ob Befruchtung crfolgt oder nicht, ob der Keimling abstirbt oder nicht, die volle ausreichende Nährstoffmenge jedem Ei von der Mutter geliefert werden muls.

Die geringe Dottermenge des menschlichen Wis reicht gerade aus, 111! diesem den selbständigen Nahrungserwerb auf der Stufe des Einzellers und des Einzellerhaufens zu ersparen und die Entwicklungshöhe eines Übergangstieres zu erreichen, welches schmarotzend sich festsetzend sich selbsttätig ernähren kann. 
Ein Weib, welches auf Fortpflanzung verzichtet, muß zwar die körperlichen Aufwendungen für die Monatsblutungen und die Ausbildung ler Eierstockbläschen trotzdem leisten (ebenso wie der Mann auch ohne gesehlechtlichen Verkehr durch Ausbildung von. Samen belastet ist), aber diese Ausgaben verschwinden gegenüber den Ausgaben der Lebewesen mit dotterreichen Eiern. Beim neuseeländischen Schnepfenstrauls beträgt das Gewicht für ein Ei etwa ein Viertel des Körpergewichtes der Mutter, welche im Vierteljahr zwei solcher Eier zu legen hat, im Laufe des Jahrs fast das eigene Körpergewicht. Die Ausbildung eines weder der Atmung noch der Ernährung dienenden Dottersackes beim Menschenkeimling legt den Gedanken an eine Dottersackernährung dor Frucht bei den Ahnenstufen des Menschen nahe. Der Übergang zu der Ausbildung schmarotzender Keimlinge erscheint als Übergang zu ciner höheren Daseinsstufe, bei welcher die Aufwendungen für die Keimhahnzellen, also für die Fortpflanzung, in immer höherem Maße zurücktreten, gegenüber der Leistung der persönlichen Lebensarbeit, die von den körperbildenden ungeschlechtlich von der Keimbahn erzeugten Zellen und der von diesen gebauten Maschine geleistet wird.

Vom Standpunkt der Mutter ist die schmarotzende Lebensweise der Frucht wirtschaftlicher als eine gleichgroße Nährstoffbeigabe zum Ei, vom Standpunkt der Frucht sind beide Arten etwa gleichwertig. Bei den Jurchen wird die gleiche Entwicklungsstufe entweder durch Frucht: wheftung oder durch Dotterzugabe zum Ei erreicht. Die Entwicklungshöhe ist vor allem abhängig von der länge der Fruchternährung, weniger von der Art und Weise der Nahrungszufuhr. Die Fruchtanheftung bietet der Frucht größeren Schutz gegenüber äußeren Gefahren und bei Warmblütlern eine größere Gleichmäßigkeit und Sicherheit der Wärmezufuhr. Das Lebendiggebären ist in dieser Hinsicht der Eiausbrütung überlegen, zumal die gleichmäßige Wärmezufuhr dem brütenden Vogel Bowegungslosigkeit auferlegt, während die Beweglichkeit der trächtigen Säugetierweibchen weit weniger behindert ist.

Die Dauer des Aufenthalts der Eier im mütterlichen Eileiter ist für einige Säugereier auf 5-10 Tage bestimmt worden. Da der menschJiche Eileiterweg eine Länge von rund 9 Zentimetern besitzt, würde sich die Geschwindigkeit der Eibewegung in der ersten Lebenswoche auf rund 17 Millionstel Zentimeter in der Sekunde berechnen.

Mit dem Anlangen des Menscheneies in der Gebärmutter endet die Vorgeschichte der menschlichen Entwicklung, und das Leben der Keimbahnzellen tritt zunächst völlig zurück hinter dem Aufbau der Leibesmaschine, für die keim Menschen die Bewahrung und Weitergabe der Keimbahnzellen nur eine von zahlreichen Aufgaben darstellt.

Die Sexualzellen Ei und Spermatozoon spiegeln in gewisser Weise Geschlechtsunterschiede wider, die wir beim Menschen bei ihren 


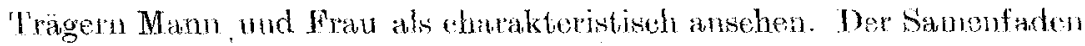
ist beweglicher, plasmaärmer, ärmer an Reserve und Nährstoffen als die Eizelle, sein Bau betont die für Bewegung geschaffenen Maschinenteile, während die Kizelle ohne Eigenbeweglichkeit durch ihren Dotter reichtum und Plasmareichtum den chemischen Teil der Leibesmaschin: betont reigt. Wie seino Samenfädon, betont auch der Körper des Mannes im ganzen mehr die für Bewegung gebauten Teile seiner Leibes:maschine, während die Reservestofflager: und dio vegetativen Teilo: weniger betont exscheinen. Das Weik exseheint wie die Hizelle mehr vegetativ betont, reservestoffrecher, in sich vollkommener und den Gatungstypus vollständiger wicdergebend, entspreahend der vollen Zahl seiner Chromosomen, während der Mamn entsprechend sejuer unvollständigen Chromosomengarnitur meht das Individuelle als daw GattungsmäBige in seiner Hoheinung ausdrückt. Wenn der Mann

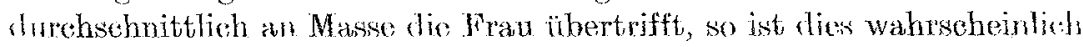
zurückzuführen auf eino gewisse Jugendlichkeit der Cattung, bei dre die Männchen zunächst in der Körpergrößc die Weibchen übertreffen. Nltert die Art, wo kommen die Weibchen den Männchen an GröBe allmäblich gleich und ïbertreffen sie schlieblich an Masse. Bei der Mehrrahl der hento lebenden 'Tierarten übertreffen die Weibehen die Männchen an Jeibosmasse. Boi den Europäers macht sich in don Kultursehichtes eine Abnahme in der Gröbendifferenz dor Geschlechter so dentich bomerkbar, dak die neugeborenen Mädchen in den reichsten Familien don Knaben an Gewieht völig groichstehen mel viele weibliche Individuen lio mämblichen an Gröbo in stets zunehmender Zahl äbertreffen. Wi: lehten vor dem Woltkrieg in dem heitalter der groben Königinnou and der kloinen. Könge, wio on scharfsinniger Beobaehter in cincy Zeitung treffend bemerkte. Es ist nieht ummöglieh, dab hei der weilsen

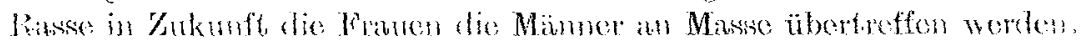

